\title{
Expanded Focus on Non-equilibrium Thermodynamics
}

\author{
Signe Kjelstrup • William M. Haynes
}

Published online: 17 July 2013

(C) Springer Science+Business Media New York 2013

The field of non-equilibrium thermodynamics is currently a subject of increasing interest. Several books on new developments and applications have appeared, providing a general thermodynamic basis for a multitude of rate processes. The classical theory has been recently extended to deal with transport through phase boundaries and to non-equilibrium quantum systems. Optimization of entropy production is important in chemical and mechanical engineering. Several lines of research are addressing the non-linear regime. These generalizations give hope for more consistent thermodynamic modeling in the future, for yet a wider range of applications.

The International Journal of Thermophysics, with a tradition for reports on high quality experimental, computational, and theoretical results in thermophysics, has therefore found it timely to accept responsibility for assisting in the development of this branch of thermophysics. By naming a new Associate Editor with special responsibility for non-equilibrium thermodynamics, we hope to give the field more attention. Our hope is that a synergy can be realized between our traditional target group of authors and a new group of authors who are experienced in developing and using non-equilibrium thermodynamics to explain, understand, and describe processes with several driving forces.

As a first move to foster such a development, this issue is publishing a collection of six articles, based on invited talks presented at the 6th International Workshop of

\footnotetext{
S. Kjelstrup $(\varangle)$

Department of Chemistry, Norwegian University of Science and Technology,

Realfagbygget, D3, 7491 Trondheim, Norway

e-mail: signe.kjelstrup@nt.ntnu.no

W. M. Haynes $(\varangle)$

National Institute of Standards and Technology, Mail Stop 647,

325 Broadway, Boulder, CO 80305-3337, USA

e-mail: william.haynes@nist.gov
} 
Non-equilibrium Thermodynamics in Røros, Norway, August 2012. The review article (Liu et al.) gives an overview of recent accomplishments enabling computation of ternary diffusion coefficients, from sets of molecular dynamics simulations which provide thermodynamic as well as transport data in facilitated ways. The work, based on the Onsager relations of classical irreversible thermodynamics, provides excellent new approaches for predictions of multicomponent diffusion. Non-equilibrium molecular dynamics simulations, techniques created to compute transport properties, are useful for systems that are difficult to manufacture and control. The article by Schnell and Vlugt provides an example of a simulation that gives detailed insight in the mechanism of transport. Chemical reactions have long been considered beyond the realm of non-equilibrium thermodynamics. This is no longer so, if we invoke internal variables, as is shown by Rubi et al. with equations derived from the mesoscopic realm. Interestingly, a cycle of chemical reactions can maintain self-assembly out of equilibrium, as explained by Koper et al. Constant dissipation of energy is then observed. Both articles on chemical reactions provide new equations for data reduction.

An important aim of applied non-equilibrium thermodynamics is to propose experiments that follow from theoretical developments. Chassagne give an exact new calculation of the polarizability of colloidal spheroids, which can be measured. The article by Osmanov and Öttinger provides another challenging example, aiming to create the important link between the world of quantum mechanics and that of macroscopic thermodynamic variables.

The editors encourage new contributions with an emphasis on results similar to the ones presented here. 